\title{
Nutritional Management of Paediatric Crohn's Disease
}

\author{
Nestlé Health Science online symposium, $4^{\text {th }}$ June 2020. \\ This was planned to be presented at the cancelled $6^{\text {th }}$ \\ World Congress of of Pediatric Gastroenterology, \\ Hepatology and Nutrition (WCPGHAN)
}

\author{
Chairperson: Paolo Lionetti ${ }^{1}$ \\ Speakers: \\ Javier Martín-de-Carpi, ${ }^{2}$ Rotem Sigall-Boneh, ${ }^{3}$ Eytan Wine ${ }^{4}$ \\ 1. Paediatric Gastroenterology and Nutrition, Meyer Children's Hospital, Florence, \\ Italy \\ 2. Gastroenterology, Hepatology and Nutrition Department, Hospital Sant Joan de \\ Déu, Barcelona, Spain \\ 3. Clinical and Research Dietitian, Wolfson Medical Center, Holon, Israel \\ 4. Department of Pediatrics, Division of Pediatric Gastroenterology \& Nutrition, \\ University of Alberta, Edmonton, Canada
}

Disclosure: $\quad$ Prof Lionetti has received speaker and advisory board fees from AbbVie, Sandoz, Pfizer, Janssen, Nestlé Nutrition, and Nutricia. Prof Martín-de-Carpi has received consultation fees, research grants, or honoraria from AbbVie, MSD, Otsuka, Adacyte, Celltrion, Kern Pharma, Janssen, Roche, Celgene, Dr Falk Pharma, Ferring, FAES, Nestlé Health Science, Abbott, Mead \& Johnson, Lactalis, Nutricia, Biogaia, Casen Recordati, Hero, Ferrer, Ordesa, Pharmes, and Zambón. Mrs Sigall-Boneh has received consulting fees from Nestlé Health Science; and speaker fees from Nestlé Health Science, Takeda, and Megapharm. Prof Wine has received honoraria from Nestlé Health Science, AbbVie, and Janssen; and funding from the Canadian Institutes of Health Research (CIHR), the Garfield Weston Foundation, IMAGINE SPOR Network, and the Crohn's and Colitis Foundation of America (CCFA).

Acknowledgements: Writing assistance was provided by Dr Eleanor Roberts, Beeline Science Communications Ltd, London, UK.

Support:

The publication of this article was funded by Nestlé Health Science. The views and opinions expressed are those of the presenters. Content was reviewed by the speakers for medical accuracy.

Citation:

EMJ. 2020;DOI:10.33590/emj/040820.

\section{Meeting Summary}

For many people with Crohn's disease (CD), onset occurs in childhood or adolescence. Treatment for CD has moved from predominantly surgical to, more often, pharmacological. While successful for many, others have tried various medications and combinations without long-term success and, for all, drug treatment needs to be balanced with potential therapy risks. Findings that diet can impact pathogenesis of CD to cause and exacerbate symptoms have inspired studies of dietary interventions. The Crohn's Disease Exclusion Diet (CDED) was developed following the observation that certain dietary components were linked to inflammation and gut dysbiosis found in those with CD. This three-phase diet included two periods of a highly-controlled and prescribed diet, followed by a 
maintenance diet in which patients had a wider choice of foods. The diet limited ingestion of foods that may trigger inflammation and/or dysbiosis in CD, such as saturated fats, wheat, carrageenan, and some dairy products, and included healthy choices, such as fruits, vegetables, lean protein sources, and complex carbohydrates. It was nutritionally balanced, science-based, and included foods that were widely accessible. Based on findings from clinical trials and case studies, four experts (Prof Lionetti, Prof Martín-de-Carpi, Mrs Sigall-Boneh, and Prof Wine) discussed the background of CD, current treatment options, the utility of dietary therapies including CDED, and how all healthcare professionals (HCP) looking after children and adolescents with CD should consider the use of diet as part of their therapy.

\section{Introduction to Nutritional Management of Paediatric Crohn's Disease}

\section{Professor Paolo Lionetti}

CD, a complex condition with a genetic predisposition, can start in childhood or adolescence.' Surgery used to be the mainstay for $C D$ but rates have now decreased, ${ }^{2}$ possibly because of the use of biopharmaceutical drugs ('biologics'), more tailored use of thiopurines, and earlier disease recognition. ${ }^{3}$ However, pharmacotherapy may be limited because each biologic targets only one pathway. As other pathways come into play leading to disease progression, it is not known if, with these agents, the natural history of $C D$ is changed in the long term. ${ }^{3,4}$ Dietary therapy with $C D$ may help in this respect.

Diet and the immune response can affect a person's gut microbiota. A diet containing cereals, legumes, and fibre, such as that consumed by children in rural Burkina Faso, promotes a gut microbiota with a wide variety of bacterial species able to extract metabolic energy from ingested plant polysaccharides. ${ }^{5}$ This leads to the production of short-chain fatty acids, which help control inflammation ${ }^{6}$ and improve epithelial cell energy metabolism in colitis. ${ }^{7}$ Comparison of these rural children to those who moved to a more urban environment found the change to a diet higher in calories and lower in fibre altered microbiota composition and lowered short-chain fatty acid levels to become similar to children in urban Italy.

However, location (rural versus urban) is less the problem than what is consumed. A 'Mediterranean diet', including fruits, vegetables, whole grains, and seafood, is associated with high microbiome diversity, an intact gut epithelial barrier, and a balanced immune function. In contrast, a 'Western diet', low in fibre and high in total fat, animal protein, polyunsaturated fatty acids, and refined sugars, is associated with alterations in microbiome composition and metabolic activity (dysbiosis), increased epithelial barrier permeability, and loss of immune system tolerance. ${ }^{9-11}$

In the question and answer (Q\&A) session, it was asked if everyone should avoid potentially inflammatory foods, such as the additive carrageenan. While there is little evidence regarding recommendations for carrageenan for the general population according to Mrs Sigall-Boneh, Prof Lionetti illustrated how animal and in vitro models have shown a significant role for dietary components in gut health. For instance, gut epithelial function and mucous layer composition can be disrupted by carrageenan, ${ }^{12}$ emulsifiers, ${ }^{13}$ and a high-fat diet. ${ }^{14}$

Prof Lionetti hypothesised that, combined with genetic factors, low-grade inflammation caused by a Western diet can lead to CD development. For example, one large 26-year epidemiological study found $C D$ risk was inversely associated with level of fruit and fibre consumption. ${ }^{15}$ As such, controlling diet in those with $C D$, such as with the CDED, may be useful in managing CD-associated inflammation. ${ }^{16}$ 


\section{Different Approaches for the Induction and Maintenance of Remission in Paediatric Crohn's Disease}

\section{Professor Javier Martín-de-Carpi}

Treatment objectives for paediatric $C D$ include achieving and maintaining remission, halting disease progression, and providing adequate nutrition for growth. There is also a focus on limiting potentially damaging medication side effects and reducing surgery and hospitalisations, along with holistic objectives, such as improving quality of life and facilitating support. Of great importance is recognising lifereducing factors associated with $C D$, including infections, disability, cancer, and bone mineral density problems. While many paediatric HCP do not see such complications, these may occur as the patient grows up with CD.

Following European Society for Paediatric Gastroenterology, Hepatology and Nutrition (ESPGHAN) and the European Crohn's and Colitis Organisation (ECCO) guidelines, first-line treatment for children with $C D$ is $6-8$ weeks of exclusive enteral nutrition (EEN). ${ }^{1718}$ Maintenance therapy may include immunosuppressant drugs, such as thiopurines or methotrexate (MTX); anti-TNFa drugs, including infliximab and adalimumab;:17,19 other biologics, such as vedolizumab or ustekinumab; or combination therapy. ${ }^{20-24}$ The benefits of drug therapy must always be considered alongside the risks.

In the long term, some people fail drug therapy at various stages and switching therapies and/ or surgery may be required. ${ }^{25-28}$ There are also those that unsuccessfully progress down each line of therapy and hit, according to Prof Martínde-Carpi, a 'non-exit road'. ${ }^{17}$ However, he stressed, treatment should not be 'one-directional' and HCP should reconsider previous treatments or re-examine those treatments not used before, including "going back to basics."

CD pharmacotherapy is utilised to stop the immune system reaction and aid the microbiota in blocking inflammation; however, these may not address environmental factors causing CD. Proper dietary interventions could address all of these needs (Figure 1).

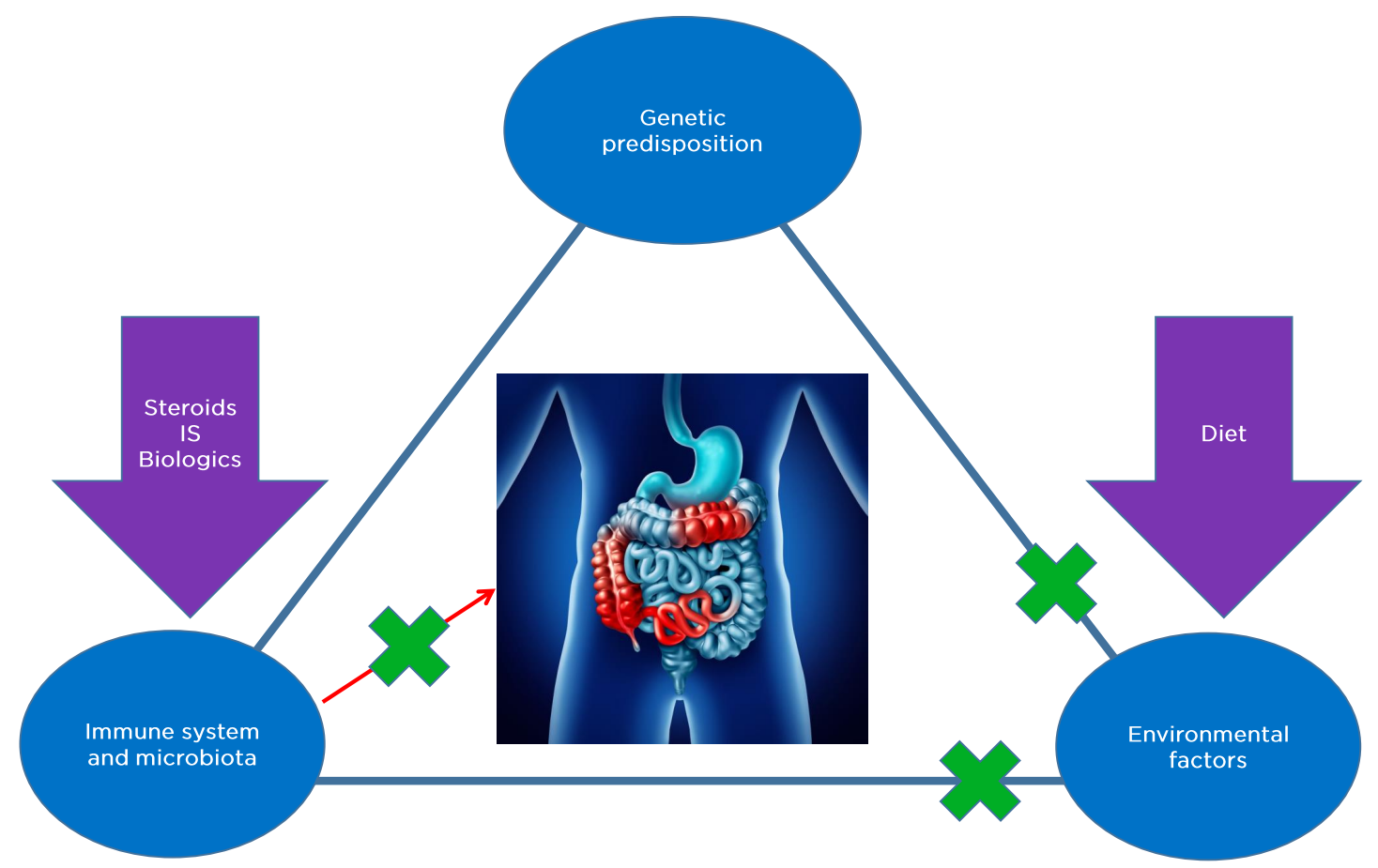

Figure 1: Crohn's disease may have a genetic factor that cannot be changed, but pharmacotherapy and diet can address factors associated with a person's immune system, microbiota, and environment.

IS: Immunosuppressant drugs. 
Paediatricians should discuss diet as part of $C D$ treatment because it is a concern for patients and many parents ask about it when their child is diagnosed. ${ }^{29}$ Some patients/parents try restrictive, unhealthy diets but, following EEN, previous advice was against dietary modification as no single food was implicated as being entirely involved in CD. ${ }^{30}$ The CDED was highlighted by Prof Martín-de-Carpi as being one that people might consider trying because it fits well with the frequent demand by patients and their relatives regarding acceptability and compliance. It is as effective as EEN in achieving clinical and biochemical remission and mucosal healing but superior to EEN in tolerance and compliance. ${ }^{31-37}$ Thus, the CDED may constitute a nutritionally balanced long-term strategy for maintaining remission if complied with adequately.

In conclusion, Prof Martín-de-Carpi emphasised how treatment plans need to be accessible and involve tools for patient autonomy to help them manage their daily life with less direct assistance from HCP. Allowing a patient to actively participate in their care could lead to better treatment adherence and lifestyle changes.

\section{Updates in the Dietary Management of Crohn's Disease with the Crohn's Disease Exclusion Diet: Can We Predict a Patient's Response?}

\section{Mrs Rotem Sigall-Boneh}

As discussed above, CD-associated microbiome changes can lead to inflammation and CD symptoms. For example, dysbiosis is associated with high fat and sugar intake, low fibre intake, and ingestion of gluten, emulsifiers, and taurine. Bacterial pathogenicity, virulence, epithelial translocation, and mucosal adhesion are also associated with many of these dietary factors, as well as with ingestion of maltodextrins, high animal protein intake, and low intake of resistant starch. ${ }^{31}$

CDED, developed in 2011 by Prof Arie Levine, is a proven dietary plan with a high level of evidence for efficacy. ${ }^{16}$ CDED excludes potentially proinflammatory dietary factors to help reduce inflammation and improve the microbiome balance. It comprises three phases defined by which foods, and how much, can be consumed in each. Phases 1 and 2 are 6 weeks each and are designed to induce remission, with Phase 3 set as a continuum with more flexibility in the diet, adapted for maintenance of remission. Progressive exposure, where at each phase more foods are allowed, makes it easier for long-term compliance. CDED is balanced in nutritional needs, is palatable, and includes allowed foods (some of which are highly recommended), excluded foods, and those that may require exclusion or reduced exposure depending on the individual. ${ }^{31}$ The diet uses foods that can be widely accessed and includes recipes and a support programme (ModuLife, Nestlé Health Science, Switzerland).

CDED foods include fruit, vegetables, resistant starch, high-quality lean protein sources, complex carbohydrates, and healthy oils. Food choices define consumption of low or no amounts of inflammatory-linked components, including animal and saturated fats, taurine, wheat, haem/ iron, emulsifiers, maltodextrins, carrageenan, sulphites, and dairy products. ${ }^{31}$ In Phase 1, CDED foods constitute approximately $50 \%$ of a patient's energy requirements, with $50 \%$ from partial enteral nutrition (PEN). In the Q\&A, Prof Lionetti discussed how tube-fed EEN was originally essential because the formula was “unpalatable," but more recent polymeric formulas may be taken orally because "the taste is much better and accepted by both adults and children." A similar formula is used as part of the CDED to complement nutrition and energy needs. Moreover, during the Q\&A, Mrs Sigall-Boneh discussed how in Phase 1, fibre is limited as a result of inflammation that might cause narrowing of the intestine and might lead to abdominal pain; however, at later stages exposure to fibre is recommended if no stricture is present. In Phase 1, refined rice is allowed if cooked with a lot of water to reduce potential arsenic exposure. In Phases 1 and 2, frozen and processed foods are discouraged because some have additives; however, some can be introduced in Phase 3 if tolerated.

CDED studies have found CDED to have better tolerance and adherence than EEN ${ }^{16,32}$ and have success in complicated situations, for example in patients who do not respond to biological therapy. ${ }^{33}$ 


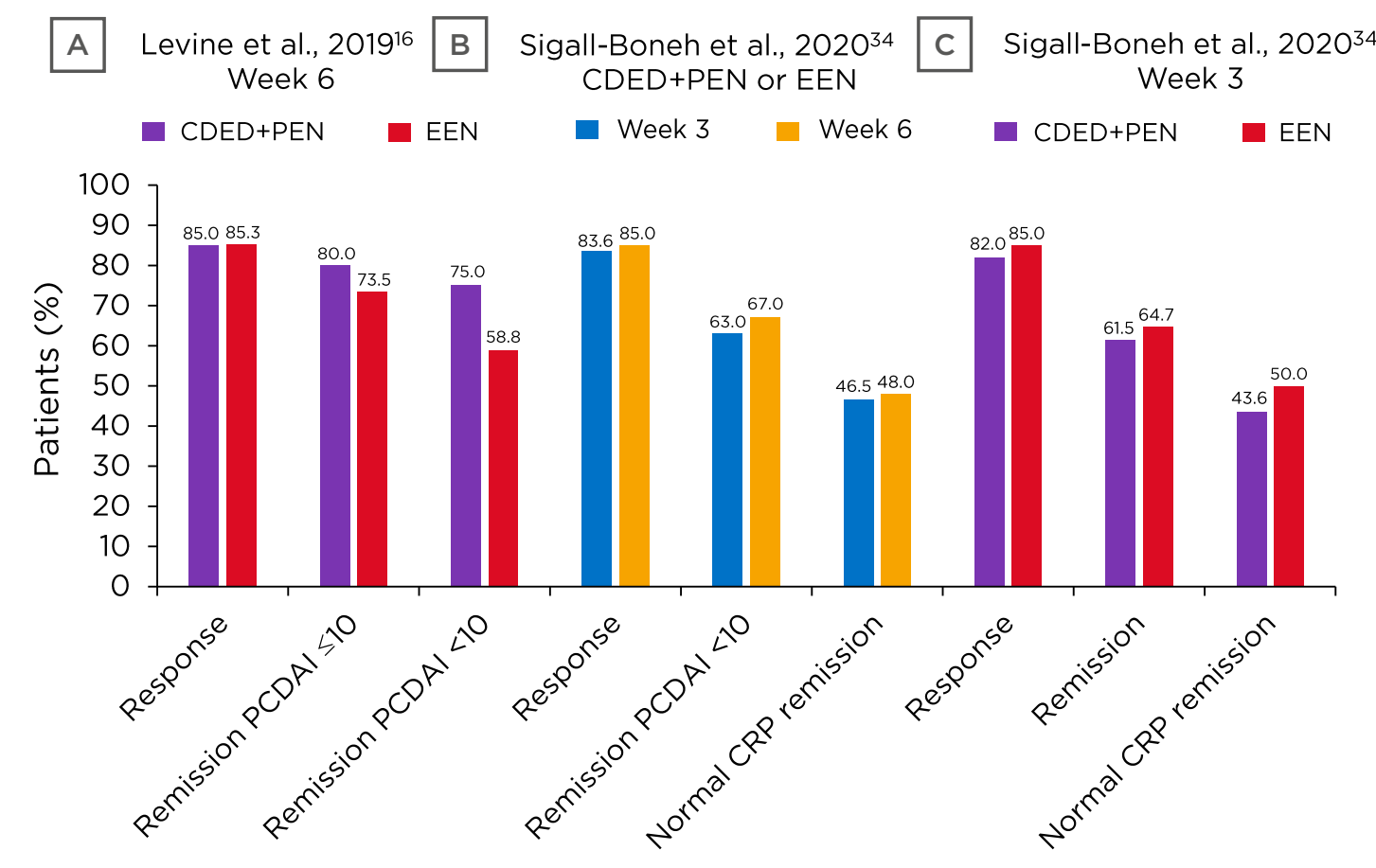

Figure 2: A) CDED+PEN at Week 6 response and remission; B) response, remission, and CRP levels following dietary therapy (CDED+PEN or EEN); C) CDED+PEN or EEN at Week 3 response, remission, and CRP levels.

CRP: C-reactive protein; CDED: Crohn's Disease Exclusion Diet; EEN: exclusive enteral nutrition; PCDAI: Paediatric Crohn's Disease Activity Index; PEN: partial enteral nutrition.

Adapted from Levine et al.;1 Sigall-Boneh et al. ${ }^{34}$

In 2019, Levine et al. ${ }^{16}$ published the first randomised controlled trial that investigated CDED+PEN $(n=40)$ compared to EEN $(n=38)$ in children aged 4-18 years with mild-to-moderate luminal CD, defined by a Paediatric Crohn's Disease Activity Index (PCDAI) score between 10 and 40. By Week 6 (Figure 2A), there was a high and comparative response rate with both diets and many participants achieved a PCDAl score $\leq 10$, with some having a PCDAl score $<10$.

As dietary treatment can lead to remission, Mrs Sigall-Boneh posited that early response might be used to predict this. An assessment of Levine et al.'s ${ }^{16}$ data found that most participants responded to dietary therapy by Week 3, with many having a PCDAI score $<10$ (Figure 2B). Similar response/ remission rates were shown between diets at both Week 3 and Week 6 (Figure 2C), along with a significant reduction in the inflammatory biomarker C-reactive protein (CRP) in both groups (Figure $2 \mathrm{~B}$ and Figure $2 \mathrm{C}$ ). ${ }^{34}$
In this analysis, $75.4 \%$ of those who achieved response at Week 3 showed remission at Week 6. Of those with a PCDAl score $<10$ at Week 6 , 94.0\% achieved response and 81.6\% achieved remission at Week 3 . As such, remission at Week 3 was found to be predictive of remission at Week 6 (odds ratio [OR]: 6.37; 95\% confidence interval [Cl]: 1.600-25.000; $\mathrm{p}=0.008$ ) and Week 12 (OR: 4.5; 95\% Cl: 1.235-16.476; $\mathrm{p}=0.023)$. Week 6 remission was negatively predicted by poor compliance (OR: 0.75; 95\% Cl: 0.012-0.460; $p=0.006){ }^{34}$

It may be that Week 3 response results can be used as a diagnostic tool such that for responders, assurance can be given that they will very likely continue to respond to CDED, potentially for the rest of their lives. If a person does not respond at Week 3, with no other biological markers indicating signs of improvement, it may indicate the need to abandon the diet and initiate other treatments instead of persisting to Week 6. 
In the Q\&A, it was suggested that response factors could include the inflammatory protein faecal calprotectin (FCP) and microbiome analysis. However, as discussed by Prof Wine, Levine et al. ${ }^{16}$ found FCP response to be slow and, while microbiome changed to a degree with dietary therapy, microbial balance predominantly reappeared when food was reintroduced to EEN, but not with Phase 2 CDED.

The Q\&A also raised the question of whether there were comparison studies regarding mucosal healing with CDED or drug therapy. The panel discussed how studies that have examined this, including a comparison of CDED with steroids, were ongoing. Prof Wine emphasised that it was important to consider not only how quickly a treatment works, as may be found with steroids, but how safe it is long term.

In conclusion, Mrs Sigall-Boneh emphasised how therapeutic strategies should be personalised and include the option of dietary modulation. She suggested that short-term use of dietary therapy may be warranted in some to identify dietary response and that dietary therapy can be used as a standalone therapy, a bridge to biological therapy, or as an adjunct to medication.

\section{From Theory to Practice: Clinical Use of Crohn's Disease Exclusion Diet in Real-Life Cases}

\section{Professor Eytan Wine}

The aforementioned clinical trial excluded children using steroids or biologics and those with perianal or primary colonic disease. ${ }^{16,35}$ Prof Wine posited that other CD cohorts could benefit from CDED, such as those with severe luminal $C D$, individuals who are refractory to drug therapy, or for those where CDED might be beneficial beyond 12 weeks. Recent studies have highlighted the important role of dietary therapies for those with $C D$ in settings different to clinical trials $^{36,37}$ and ongoing studies are focussing on more severe patients.

\section{Case Presentations}

\section{Case 1}

Case 1 highlighted the utility of CDED+PEN for severe $C D$. A 12-year-old male with a genetic predisposition experienced escalating pain over 3 months, bloody diarrhoea, weight loss, mouth sores, and fatigue. His PCDAI was 45 and he had high inflammatory markers including CRP (24.5 mg/L) and FCP $(3,378 \mu \mathrm{g} / \mathrm{g})$ at diagnosis. Endoscopy revealed panenteric disease and biopsies were positive for granulomas (indicating immune system activation and inflammation). $C D$ was severe in the terminal ileum where ultrasound revealed a long, thickened segment $(3.5-4.0 \mathrm{~mm})$ with fat proliferation and stratification loss.

Two weeks of EEN achieved almost complete remission with no pain, little diarrhoea, normal CRP ( $<0.5 \mathrm{mg} / \mathrm{L}$ ), and a PCDAI of 10 . While usual practice would be to continue EEN, the patient undertook Phase 1 CDED+PEN and was happy to resume eating at least some food. Complete clinical remission was achieved at 6 weeks, with a PCDAI of O, normal CRP (0.2 $\mathrm{mg} / \mathrm{L})$, and normal terminal ileum thickness (0.9-1.4 mm). After 6 weeks of Phase 2 CDED, PCDAl was O, CRP was <0.2 mg/L, and FCP was $229.4 \mu \mathrm{g} / \mathrm{g}$. The patient continued onto Phase 3 maintenance CDED and remained in remission 22 weeks after initial treatment.

Reflecting this case, one Q\&A query posited whether CDED could be used as maintenance therapy alone. Data are currently only based on experience, though trials are ongoing. Studies of maintenance CDED are "challenging," explained Mrs Sigall-Boneh, "as patients can have some 'free' meals and choose what to eat. We educate the patient to maintain some kind of restrictive diet but still go out and enjoy a normal life and find the balance." Prof Wine added that: "There is some fatigue for patients on a maintenance diet even though it's more liberal. There are limitations and certainly for some children it's more difficult while some love it."

During the Q\&A, Prof Wine shared: "Even if we're getting a partial response [with CDED] and you get along with less adjuvant therapy, that's an accomplishment. If you can get the effect you get with EEN of not needing steroids and allowing us to complete the workup and start the patient 
on another therapy in a better situation, that's a good enough reason to do it." The following cases highlight this.

\section{Case 2}

Case 2 examined CDED+PEN in someone who had lost response to biologic treatment. This 22-year-old male was diagnosed with CD aged 16 years old and was treated with infliximab plus MTX, then switched to adalimumab plus MTX when infliximab response was lost. He achieved clinical remission including partial mucosal healing; however, there was noticeable loss of response to adalimumab when MTX was discontinued.

On presentation, the patient reported frequent diarrhoea, weight loss, anorexia, and resumption of symptoms over 6 weeks. Investigations revealed elevated CRP (19 $\mathrm{mg} / \mathrm{L}$ ) and FCP (604 $\mu \mathrm{g} / \mathrm{g}$ ). With adalimumab retained, the patient was given Phase 1 CDED+PEN. By 6 weeks, his CRP level normalised, he had no pain or diarrhoea, and was nearing remission so he proceeded to Phase 2. Week 12 assessment showed reduced FCP (228 $\mu \mathrm{g} / \mathrm{g})$ and normal CRP. Unfortunately, as a student away from home, while he continued taking adalimumab, he could not maintain the diet and he relapsed. Importantly, this case showed that dietary intervention can be successful in addition to biologics, as supported by similar case reports, ${ }^{3}$ and highlighted the importance of compliance in achieving effectiveness of dietary management of CD.

\section{Case 3}

Case 3 discussed refractory disease with an indication for surgical intervention. A 15-year-old female was diagnosed with severe panenteric CD aged 5 years old. She was initially treated with infliximab plus azathioprine (later switched to MTX), then switched to adalimumab plus MTX following loss of response. Unfortunately, her symptoms, including abdominal pain, vomiting, and diarrhoea, were ongoing and she was hospitalised with a severe relapse. At this point, she showed severe panenteric stricturing, inflammation (an erythrocyte sedimentation rate of $90 \mathrm{~mm} /$ hour), and adalimumab trough levels of $15 \mu \mathrm{g}$, suggesting pharmacodynamic failure.
Remission was achieved after 2 weeks EEN and she commenced 12 weeks CDED+PEN (Phases 1 and 2). While this therapy brought remission, she felt it was too difficult to continue with. Ustekinumab therapy was initiated but she was nonresponsive after 6 months and was hospitalised. Examination revealed numerous deep ulcers from rectum to caecum: a $15 \mathrm{~cm}$ thickened bowel loop with strictures and proximal loop inflammation (total $30 \mathrm{~cm}$ ). She had an erythrocyte sedimentation rate of $76 \mathrm{~mm} / \mathrm{hour}$ and indications of microcytic anaemia.

This patient had a clear indication for surgery, including resection plus ileostomy, but she consented to retrying dietary therapy with EEN, followed by 12 weeks of CDED+PEN, to try to avoid surgery. Colonoscopy following therapy showed no inflammation with a normal colon, though because the ileum could not be intubated, a small segment was removed. Following surgery, adalimumab was restarted, and the patient remained in remission after 2 years.

During the Q\&A, adalimumab re-administration was queried because the patient had previously lost response to it. Prof Wine explained that: "This treatment was the most effective over time. The rationale was that the diet and removal of the strictured segment would lead to a debulking effect, get inflammation under control, and get the patient into remission to set them up to have more success with the treatment they were on before." Further, it was asked what could be done if a symptom flare-up occurred during Phase 3 maintenance CDED. Case 3 highlighted how, even without maintenance therapy, a person can return to Phase 1 CDED and it can be used alongside drug therapies to help induce and retain remission.

Prof Wine concluded by suggesting that there are many people with CD for whom CDED can be used at any disease stage or location, as shown in Figure 3.

To highlight one example of a potential CDED candidate, the Q\&A considered people with strictures. Prof Wine discussed how CDED Phase 1 is low in fibre because of concern from stricturing disease and those with stricturing disease were excluded from the RCT ${ }^{16}$ as likelihood of success was lower. 


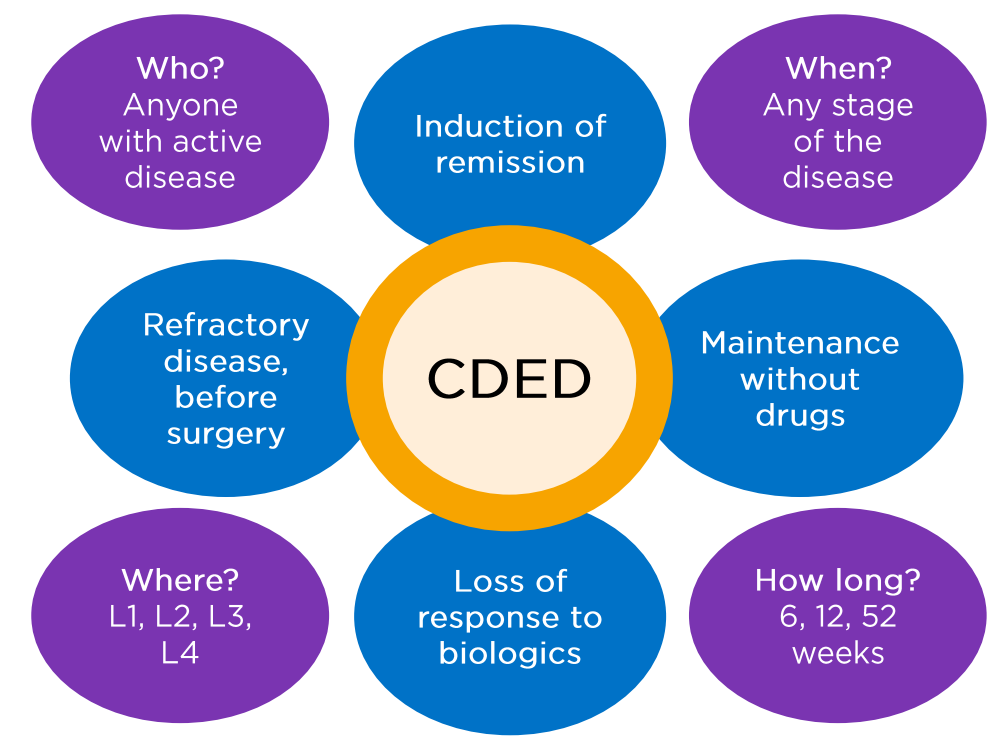

Figure 3: Who is Crohn's disease exclusion diet therapy for?

CDED: Crohn's Disease Exclusion Diet.

Adapted from Prof Arie Levine, personal communication.

Prof Martín-de-Carpi agreed that these patients "may not be the best candidate for CDED; however," he continued, "if there is a long delay in diagnosis and clear MRI images of strictures and previous dilations, it's reasonable to try CDED or EEN... [with] close follow-up to try and get a full response." Although, he advised that: "If you don't hit the target you're looking for, change to a treatment that's more effective."

Prof Lionetti discussed how there can be problems discriminating between inflammation and fibrosis. However, he posited that a liquid diet could help prior to surgery to limit strictures. This position was endorsed by Mrs Sigall-Boneh, whose patients often have EEN for 1-2 weeks prior to surgery. Prof Wine shared that: "There are some [with strictures] l'd try with a liquid diet in the first week or so and, if we see improvement, we would use CDED to try and settle inflammation." This has the advantage of avoiding medicationrelated side effects. Prof Martín-de-Carpi agreed that: "We all have some patients you think are going to require surgery, but you can control the disease and diminish inflammation so it's worth trying." Prof Wine reported that there are, however, "some cases where we don't hesitate to go to surgery or use biologics."

\section{Conclusion}

While pharmacotherapy for $C D$ has reduced the need for surgery, there are concerns for the utility of single-target medications and adverse event profiles. Dietary therapy, such as with CDED, is efficacious for many and could be trialled for a wide range of patients.

WATCH THE FULL ONLINE SYMPOSIUM ON DIETARY MANAGEMENT OF PAEDIATRIC CROHN'S DISEASE https://www.nestlehealthscience.com/newsroom/events/online-symposium-dietary-managementpediatric-crohn-disease 


\section{References}

1. Ruel $\mathrm{J}$ et al. IBD across the age spectrum: is it the same disease? Nat Rev Gastroenterol Hepatol. 2014;11(2):88-98.

2. Frolkis $A D$ et al. Risk of surgery for inflammatory bowel diseases has decreased over time: a systematic review and meta-analysis of population-based studies. Gastroenterology. 2013;145(5):9961006

3. Dubinsky M. Have we changed the natural history of pediatric Crohn's disease with biologics? Dig Dis. 2014;32(4):360-3.

4. Kugathasan S et al. Prediction of complicated disease course for children newly diagnosed with Crohn's disease: a multicentre inception cohort study. Lancet. 2017;389(10080):1710-8.

5. De Filippo $\mathrm{C}$ et al. Impact of diet in shaping gut microbiota revealed by a comparative study in children from Europe and rural Africa. Proc Natl Acad Sci U S A. 2010;107(33):14691-6.

6. Ahmad MS et al. Butyrate and glucose metabolism by colonocytes in experimental colitis in mice. Gut. 2000;46(4):493-9.

7. Venkatraman A et al. Amelioration of dextran sulfate colitis by butyrate: role of heat shock protein 70 and NFkappaB. Am J Physiol Gastrointest Liver Physiol. 2003;285(1):G177-84.

8. De Filippo $\mathrm{C}$ et al. Diet, environments and gut microbiota. A preliminary investigation in children living in rural and urban Burkina Faso and Italy. Front Microbiol. 2017:8:1979.

9. Khalili $\mathrm{H}$ et al. The role of diet in the aetiopathogenesis of inflammatory bowel disease. Nat Rev Gastroenterol Hepatol. 2018;15(9):525-35.

10. Martinez-Medina M et al. Western diet induces dysbiosis with increased E coli in CEABAC10 mice, alters host barrier function favouring AIEC colonisation. Gut. 2014;63(1):116-24.

11. Agus A et al. Western diet induces a shift in microbiota composition enhancing susceptibility to adherentinvasive $E$. coli infection and intestinal inflammation. Sci Rep. 2016;6:19032.

12. Fahoum $L$ et al. Digestive fate of dietary carrageenan: evidence of interference with digestive proteolysis and disruption of gut epithelial function. Mol Nutr Food Res. 2017;61(3):1600545.

13. Chassaing $B$ et al. Dietary emulsifiers impact the mouse gut microbiota promoting colitis and metabolic syndrome. Nature. 2015;519(7541):926.
14. Gruber L et al. High fat diet accelerates pathogenesis of murine Crohn's disease-like ileitis independently of obesity. PLoS One. 2013;8(8):e71661.

15. Ananthakrishnan $\mathrm{AN}$ et al. A prospective study of long-term intake of dietary fiber and risk of Crohn's disease and ulcerative colitis. Gastroenterology. 2013;145(5):970-7.

16. Levine A et al. Crohn's disease exclusion diet plus partial enteral nutrition induces sustained remission in a randomized controlled trial. Gastroenterology. 2019;157(2):44050.e8.

17. Ruemmele FM et al. Consensus guidelines of ECCO/ESPGHAN on the medical management of pediatric Crohn's disease. J Crohns Colitis. 2014;8(10):1179-207.

18. Ashton JJ et al. Exclusive enteral nutrition in Crohn's disease: evidence and practicalities. Clin Nutr. 2019;38(1):80-9.

19. Walters TD et al. Increased effectiveness of early therapy with anti-tumor necrosis factor-alpha vs an immunomodulator in children with Crohn's disease. Gastroenterology. 2014;146(2):383-91.

20. Ledder $\mathrm{O}$ et al. Vedolizumab in paediatric inflammatory bowel disease: a retrospective multi-centre experience from the Paediatric IBD Porto Group of ESPGHAN. J Crohns Colitis. 2017;11(10):1230-7.

21. Olbjørn $\mathrm{C}$ et al. Combination of biological agents in moderate to severe pediatric inflammatory bowel disease: a case series and review of the literature. Paediatr Drugs. 2020;DOI:10.1007/s40272-02000396-1. [Epub ahead of print].

22. Assa A et al. Proactive monitoring of adalimumab trough concentration associated with increased clinical remission in children with Crohn's disease compared with reactive monitoring. Gastroenterology. 2019;157(4):985-96.e2.

23. El-Matary $W$ et al. Higher postinduction infliximab serum trough levels are associated with healing of fistulizing perianal Crohn's disease in children. Inflamm Bowe Dis. 2019;25(1):150-5.

24. Gofin $Y$ et al. Therapeutic drug monitoring increases drug retention of anti-tumor necrosis factor alpha agents in pediatric patients with Crohn's disease. Inflamm Bowel Dis. 2019;izz257. [Epub ahead of print]

25. Niv Y. Hospitalization of patients with Crohn's disease: a systematic review and meta-analysis. Isr Med Assoc J. 2020;22(2):111-5.

26. Aniwan S et al. Epidemiology, natural history, and risk stratification of Crohn's disease. Gastroenterol Clin North Am. 2017;46(3):463-80.

27. de Bie $\mathrm{Cl}$ et al. The duration of effect of infliximab maintenance treatment in paediatric Crohn's disease is limited. Aliment Pharmacol Ther. 2011;33(2):243-50.

28. de Bruyn JC et al. Long-term outcomes of infliximab use for pediatric Crohn disease: a Canadian multicenter clinical practice experience. J Pediatr Gastroenterol Nutr. 2018;66(2):268-73.

29. Wong AP et al. Use of complementary medicine in pediatric patients with inflammatory bowel disease: results from a multicenter survey. J Pediatr Gastroenterol Nutr. 2009;48(1):55-60.

30. Limdi JK et al. Dietary practices and beliefs in patients with inflammatory bowel disease. Inflamm Bowel Dis. 2016;22(1):164-70.

31. Levine A et al. Evolving role of diet in the pathogenesis and treatment of inflammatory bowel diseases. Gut. 2018;67(9):1726-38.

32. Sigall-Boneh $\mathrm{R}$ et al. Partial enteral nutrition with a Crohn's disease exclusion diet is effective for induction of remission in children and young adults with Crohn's disease. Inflamm Bowel Dis. 2014:20(8):135360.

33. Sigall Boneh R et al. Dietary therapy with the Crohn's disease exclusion diet is a successful strategy for induction of remission in children and adults failing biological therapy. J Crohns Colitis. 2017;11(10):1205-12.

34. Sigall Boneh R et al. Dietary therapies induce rapid response and remission in pediatric patients with active Crohn's disease. Clin Gastroenterol Hepatol. 2020;S1542-3565(20)30487o. [Epub ahead of print].

35. Levine A, Wine $E$. Effects of enteral nutrition on Crohn's disease: clues to the impact of diet on disease pathogenesis. Inflamm Bowel Dis. 2013;19(6):1322-9.

36. Levine A et al. Dietary guidance from the International Organization for the Study of Inflammatory Bowel Diseases. Clin Gastroenterol Hepatol. 2020;18(6):1381-92.

37. Levine A et al. A case-based approach to new directions in dietary therapy of Crohn's disease: food for thought. Nutrients. 2020;12(3):880. 